PAPER

\title{
Playability of electric snare drum based on the rebound feature
}

\author{
Masanobu Miura* \\ Faculty of Science and Technology, Ryukoku University \\ (Received 30 March 2011, Accepted for publication 22 November 2011)
}

\begin{abstract}
The playability of various snare drums has been investigated. Although snare drums are very popular, the differences in their playability have not been reported. Here, the motions in performances using an acoustic snare drum (AD) and three electronic snare drums (ED1, ED2, and ED3) were analyzed, and the relationship between the characteristics of rebound and electromyograms (EMG) on the players' arms was investigated. The rebound coefficient as the characteristic of rebound was used to obtain the difference in the drumhead for each snare drum. Ten drummers (five professional and five intermediate players) were asked to play single strokes in sixteen measures under five different tempi. Surface EMG signals from their performances on four different snare drums were recorded. Drummers were instructed to play the drums under two different strengths of 40 and $70 \%$ of their maximum EMGs by observing their EMGs in real time. (Our system was used to display EMGs.) Surface EMG signals of the flexor carpi ulnaris muscle and extensor carpi ulnaris muscle of both arms were then measured. EMG signals upon playing ED1, ED2, and ED3 were compared with those for playing AD. Obtained EMGs of intermediate players playing the electric drum with the highest rebound coefficient were found to be stronger than those for playing the acoustic snare drum, whereas those of professional were found to be less, implying that only professional drummers can take advantage of the rebound feature to suppress the physical load when drumming.
\end{abstract}

Keywords: Electromyogram, Drums, Performance movement, Characteristic of rebound, Playing strength

PACS number: 43.75.St [doi:10.1250/ast.33.170]

\section{INTRODUCTION}

Performances of several musical instruments have recently been investigated by using biological techniques [1-3]. In an investigation into drumming [1], motions were observed for not only professional but also intermediate drummers, showing that only professional drummers suppress physical loads to the arms when drumming. Moreover, to achieve such performances, they usually take the features of rebound and inertia of drumsticks into account. From the results, we could see that the features of rebound and drumstick may affect the drumming. In this investigation [1], we compared several drumsticks but used only a specific snare drum, so the drumming using various snare drums was not investigated. Moreover, the differences in the features of rebound have not yet been investigated.

The acoustic snare drum is much more widely used than the electric one. The acoustic snare drum is popular across many kinds of playing styles: pop, jazz, rock, orchestral, and wind orchestra, for example. The electric

*e-mail: miura@mail.ryukoku.ac.jp snare is a recent invention [4,5]. Unlike the acoustic snare drum, electric snare drums are played by using a sensor for drumming on the drumhead, so that the time and intensity of the drummer's strokes are correctly detected, and then, the data are fed into a sound module, generating drum-like sounds. The playback of electric drum sound can be controlled. Also, the electric snare drum can be played silently while using headphones to listen, so the players can enjoy it whenever and wherever they want. The electric snare drum is being improved to achieve the quality of the sense of drumming $[4,5]$. The electric snare drum is quite different from the acoustic snare drum in terms of the difference in constitution and drumhead material. Different drumhead materials have different elasticities, which affect the rebound of the drumstick when drumming. In addition, different constitutions have different amounts of drumhead displacement, which affects the motion of the drumstick. In this study, the difference in the motion of sticks due to the difference in constitution or material is called the "rebound feature."

By avoiding physical load when drumming, players may achieve extensional motion, which is thought to be 
important for enabling drummers to play as they want. In summary, the rebound feature affects the physical load that strongly correlates with the playability of drums. In this study, we investigate how the rebound feature affects the playability of drums.

Several types of information can be used to analyze drumming, such as the acoustic signal, motion capture data, and/or physiological data. Physiological-data-based analysis will provide the time or the amount of muscle strain. The drumstick is controlled by the motion of the arm, so by measuring the arm motion we can analyze the details of playing. Because the electromyogram (EMG) can reveal the amount of muscle activity for the intended motion, the EMG is appropriate for analyzing the performance. It is proportional to the amount of muscle activity [6] and can be used to measure the amount of arm strain.

In this study, we measure the EMG of players' arms during drumming as the index of physiological data of physical loads. By measuring the EMG of several arm parts during drumming, we can clarify the physical load.

\section{METHOD}

\subsection{Style of Drumming to Be Analyzed}

To play the drums, drummers usually use both arms and legs, so they must control both arms and legs simultaneously. More specifically, drummers need to control correctly the drumsticks and pedals, so they need to control their own arms and legs. Arms are usually used more than legs, so drummers must control their arms when drumming to play without any mistakes.

Thus, our study aims at analyzing only the arms. A famous style of practicing "rudiment" is a basic training program of drumming skill [7]. In this program, the simplest way of playing is the "single stroke," in which a drummer hits a drum with drumsticks. Figure 1 shows a musical score where " $R$ " and " $L$ " represent the right and left hands, respectively.

\subsection{Outline of Investigation}

The effect of the rebound feature on the motion of a drumstick may differ depending on the interstroke-interval (ISI) and the intensity of a single stroke. Specifically, drummers need to suppress the rebounding drumstick when playing under a long ISI, whereas less suppression is needed under a short ISI because to take advantage of it in the preparation of motion for the next stroke. In other words, under a short ISI, drummers can control drumsticks by taking advantage of their rebound. The conceptual flow of the motion and force of a drumstick is shown in Fig. 2.

When drummers play under a long ISI, the amount of muscle strain caused by the rebounding drumstick is expected to be greater than those under a short ISI. On the other hand, when drummers play a drum with high rebound

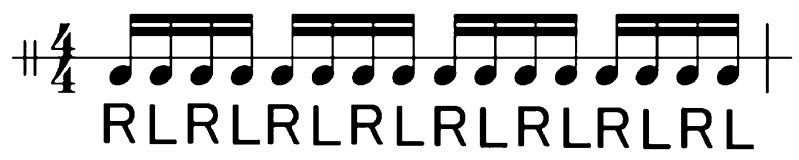

Fig. 1 Example of musical score for single stroke.

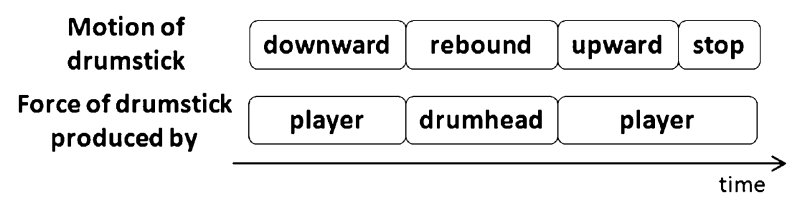

Fig. 2 Motion of drumstick and force of drumstick for a single stroke.

under a short ISI, they need to hold up the drumstick using its rebounding force much more than when using a snare drum with less rebound, so the EMG signal for holding up the drumstick of a snare drum with high rebound is expected to be weak. For drummers playing under high intensity, the amount of rebounding forces is expected to be greater than those under a force with less rebound, so the stroke intensity affects the control of the rebounding drumstick.

Therefore, we examine various types of rebound features with various tempi and stroke intensities to measure the EMG of players' arms, in order to investigate the relationship between the rebound feature and motion of drumming, to clarify how the rebound feature affects the motion of drumming.

\section{INVESTIGATION OF REBOUND FEATURE OF SNARE DRUM}

\subsection{Purpose}

The constitution of acoustic snare drums is quite different from those of electric snare drums. More specifically, the electric snare drums are of various constitutions and materials. As described above, the different constitutions and materials produce different rebound features. A previous study [1] showed that the existence of rebounding force affects the physical load of drumming, so the rebound feature of the drumhead is also expected to affect the physical load of drumming. Some of the physical indexes corresponding to the rebound features are the rebound coefficient and length of time for the shift of drumhead. The rebound coefficient means the ratio of kinetic momenta before and after hitting, and the time of sinking is represented as the required time between hitting and rebounding. The degree of sinking of the drumhead is the amount of sinking when hitting the drumhead.

In this study, we investigate the snare drum playability, which is represented as the amount of rebound force. To measure the rebound feature, the rebound coefficient is introduced as the index of the rebound strength, so that the 
Table 1 Snare drums used in this investigation.

\begin{tabular}{|c|c|c|c|}
\hline $\begin{array}{c}\text { Type of } \\
\text { snare drum }\end{array}$ & Name & $\begin{array}{l}\text { Material of } \\
\text { drumhead }\end{array}$ & $\begin{array}{c}\text { Shape of } \\
\text { drumhead }\end{array}$ \\
\hline $\begin{array}{l}\text { Acoustic } \\
\text { drum }\end{array}$ & $\begin{array}{c}\text { Acoustic snare } \\
\text { drum (AD) }\end{array}$ & $\begin{array}{l}\text { Polyester } \\
\text { film }\end{array}$ & \multirow{2}{*}{ Film } \\
\hline \multirow{3}{*}{$\begin{array}{l}\text { Electric } \\
\text { drum }\end{array}$} & $\begin{array}{l}\text { Electric snare } \\
\text { drum } 1 \text { (ED1) }\end{array}$ & Mesh & \\
\hline & $\begin{array}{l}\text { Electric snare } \\
\text { drum } 2 \text { (ED2) }\end{array}$ & Rubber pad & \multirow{2}{*}{ Pad } \\
\hline & $\begin{array}{l}\text { Electric snare } \\
\text { drum } 3 \text { (ED3) }\end{array}$ & Silicon pad & \\
\hline
\end{tabular}

coefficient is expected to correlate with the physical load of drumming [1]. Afterwards, the rebound coefficient is introduced as the index of the rebound feature. Using this coefficient, we investigate the rebound features of several snare drums, with the aim of clarifying the physical description of the differences in constitution and materials.

\subsection{Method of Experiment}

In this investigation, we used an acoustic snare drum and three electric snare drums. The rebound coefficient for each snare drum was measured. The snare drums are listed in Table 1. The electric snare drum, labeled ED1, has a film drumhead so that it gives a similar feeling to that of the acoustic drum when drumming. On the other hand, those labeled ED2 and ED3 has a pad drumhead, which is usually employed for practicing and gives a feeling far different from that of the acoustic drum. On ED1, the drumhead material is mesh so that the sound of hitting when drumming can be muffled. The rubber on ED2 is typical for many electric drums and it has been widely accepted by many drummers. The silicon pad on ED3 has appeared recently and it exhibits good rebounding characteristics.

An iron ball was magnetically controlled so that it fell down vertically onto the drumhead of each drum. The velocity of falling and bouncing upward was measured using a high-speed camera (2,000 fps). The configuration of this measurement is shown in Fig. 3, and the flow of measurement is explained below. First, an iron ball $8 \mathrm{~mm}$ in diameter was set up to $300 \mathrm{~mm}$ above the drumhead and dropped onto the drumhead. The velocity of falling and bouncing up was then measured. The time required for the iron ball to fall $50 \mathrm{~mm}$ onto the drumhead is called $T_{\mathrm{a}}$, and the time taken to rise $50 \mathrm{~mm}$ after hitting the drumhead is called $T_{\mathrm{b}}$. From the pictures taken with the high-speed camera, $T_{\mathrm{a}}$ and $T_{\mathrm{b}}$ were obtained. The obtained $T_{\mathrm{a}}$ and $T_{\mathrm{b}}$ were used to calculate the rebound coefficient $e$ by

$$
e=\frac{T_{\mathrm{a}}}{T_{\mathrm{b}}}
$$

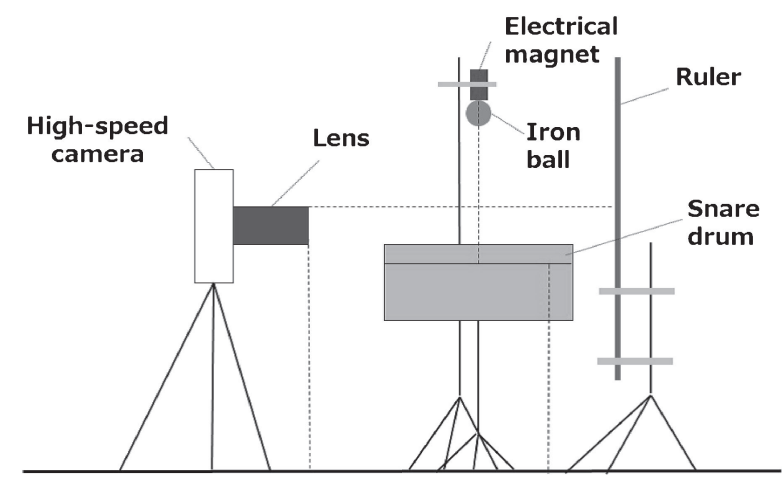

Fig. 3 Setup of measurement of the rebound coefficient.

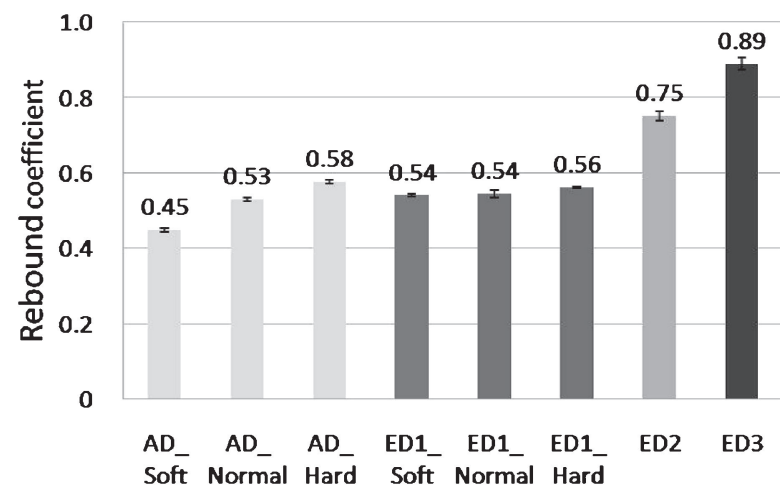

Fig. 4 Average rebound coefficient.

For all snare drums, the iron ball was dropped onto the drumhead five times and the average of obtained $e$, called $\bar{e}$, was then used. The rebound coefficients of the acoustic drum (AD) and ED1 differ owing to the straining condition of the drumhead, so the following three conditions were introduced: hard (AD_hard, ED1_hard), meaning the hardest usable performance conditions; soft (AD_soft, ED1_soft), meaning the softest usable performance conditions; and normal (AD_normal, ED1_normal) meaning standard performance conditions. All the conditions were set by a professional percussion player, and the average rebound coefficient was obtained for each snare drum.

\subsection{Results}

The obtained results are shown in Fig. 4, where the average rebound coefficient is seen to differ among snare drums. Moreover, the differences in the rebound coefficients between $\mathrm{AD}$ and ED1 or between ED2 and ED3 are relatively small compared with the differences in constitution (between film and pad). Another finding is that the range of differences in the rebound coefficient for $\mathrm{AD}$ is larger than that of ED1. Specifically for the normal condition, the order of the coefficient of rebound is AD_normal, ED1_normal, ED2, and ED3, where the normal condition is employed for $\mathrm{AD}$ and $\mathrm{ED} 1$. 


\section{APPROPRIATENESS OF THE USAGE OF EMG FOR INVESTIGATING THE MOTION OF DRUMMING}

\subsection{Purpose of Investigation}

As described in Sect. 1, the playability of drums is represented by the physical load during drumming. The physical load of arm motion can be represented by the EMG. The EMG can represent the muscle strain, so it can be used in analyzing motor control. To use the EMG to analyze drumming, we investigated the part of the muscle correlated to drumming. Here, we try to clarify the parts of muscles on the basis of the results of a previous study of EMG, and by confirming the response of the EMG to those previously defined parts, we will discuss the appropriateness of using the parts to analyze drumming.

4.1.1. Controlling drumstick for single stroke

When investigating the physical load of drumming, we must determine the part to be measured by the following relationship between the motion of arms and the activity of muscle previously defined [8]. The motion of arms can be described by yawing and pitching; rolling is expected to be correlated with stroking by the regular grip of the left hand. Yaw movement of arms is achieved by ulnar flexion and radial flexion and pitch movement by volar flexion and dorsal flexion. Therefore, the motion of arms can be achieved by the following four motions: ulnar, radial, volar, and dorsal flexion. Here the relationships of among muscle usage when carrying out the four motions are described as well as those for drumming. Figure 5 shows the direction of the four motions, and Fig. 6 shows the parts of muscle to be measured.

(a) Ulnar flexion and volar flexion

Figures 5(a) and 5(b) outline ulnar flexion and volar, respectively. In general, for ulnar flexion of the arms, we need to strain our flexor carpi ulnaris and extensor carpi ulnaris muscles, and for volar flexion, we need to strain our flexor carpi ulnaris and flexor carpi radialis muscles [8]. The positions of the flexor carpi ulnaris, extensor carpi ulnaris, and flexor carpi radialis muscles are shown in Figs. 6(a), 6(b), and 6(c), respectively.

(b) Dorsal flexion and radial flexion

Figures 5(c) and 5(d) outline dorsal flexion and radial flexion, respectively. In general, for dorsal flexion, we need to strain our extensor carpi ulnaris and extensor carpi radialis muscles, and for radial flexion, we need to strain flexor carpi radialis and extensor carpi radialis muscles [8]. The position of the extensor carpi radialis muscle is shown in Fig. 6(d).

(c) Use of muscles in each of the four motions

In each of the four motions, we need to strain flexor carpi ulnaris, extensor carpi ulnaris, flexor carpi

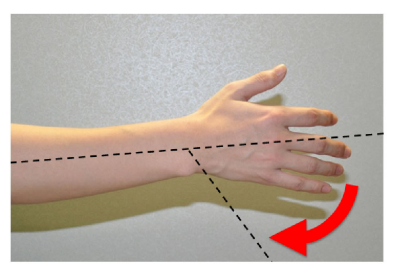

(a) Ulnar flexion

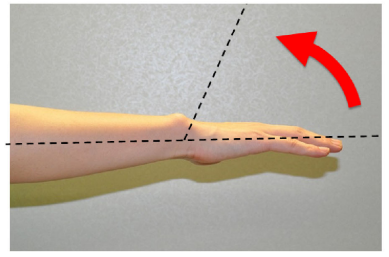

(c) Dorsal flexion

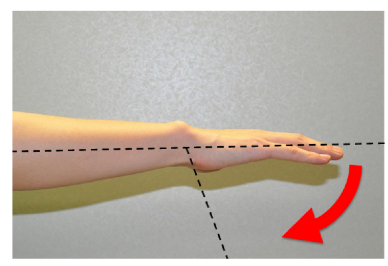

(b) Volar flexion

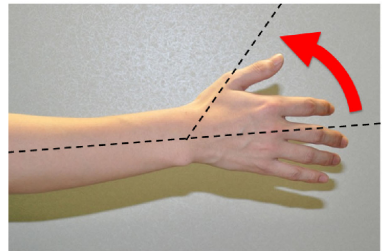

(d) Radial flexion
Fig. 5 Directions of arms for ulnar, radial, volar, and dorsal flexion.

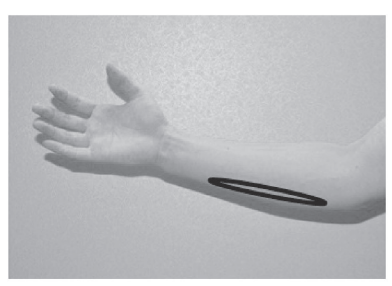

(a) Flexor carpi

ulnaris muscle

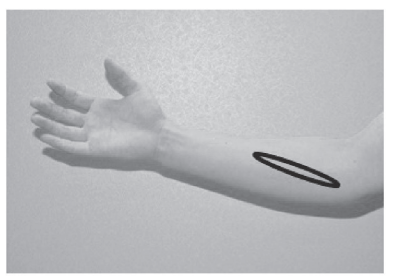

(c) Flexor carpi

radialis muscle

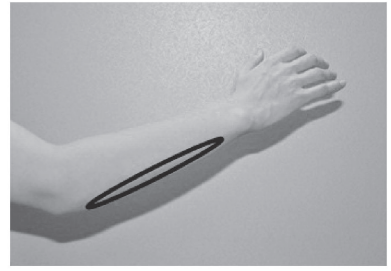

(b) Extensor carpi ulnaris muscle

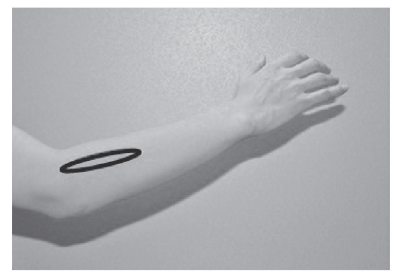

(d) Extensor carpi radialis muscle
Fig. 6 Muscle parts for measuring EMG signals.

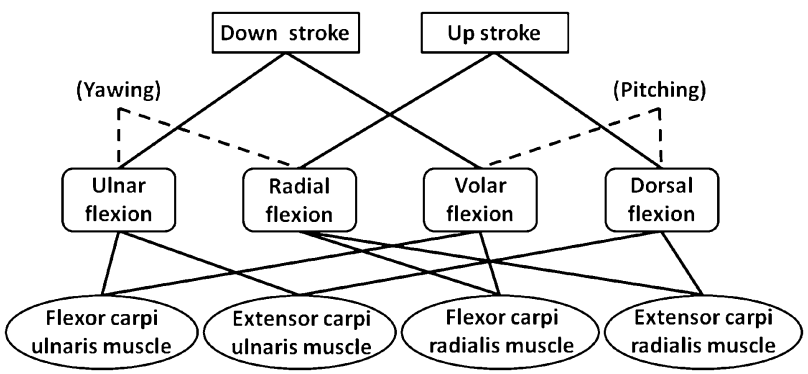

Fig. 7 Corresponding relationship for each motion and muscle part [8].

radialis, and/or extensor carpi radialis muscles. The relationships between the four motions and the control of the drumstick are shown in Fig. 7, where the relationships between the motions of arms and the four muscle motions are shown as dotted lines, and 
those between the motions of the drumstick and arm muscles are shown as solid lines.

(d) Relationship between the four muscle motions and drumming

During drumming, the drummer strokes the drumstick down by ulnar flexion and volar flexion. Also, he/she must control the drumstick after hitting the drumhead, such as pressing the drumstick onto the drumhead. In other words, the player needs to perform ulnar flexion and volar flexion to give force to the drumhead through the drumstick. In this paper, the motion of pressing the drumstick on the drumhead is called "rebound control of the drumstick." Therefore, the muscles at work for stroking down and bringing up the drumstick are the flexor carpi ulnaris, extensor carpi ulnaris, and flexor carpi radialis muscles. After hitting the drumhead, the player must hold up the drumstick and return it to its initial position. To accomplish this, the drummer must perform dorsal flexion and radial flexion of his/her arms. Therefore, the muscles used when bringing up the drumstick are the extensor carpi ulnaris, flexor carpi radialis, and extensor carpi radialis muscles.

4.1.2. Motion of drumsticks used in investigation

As described in Sect. 4.1.1, the flexor carpi ulnaris, extensor carpi ulnaris, and extensor carpi radialis muscles are useful in analyzing the down stroke of drumsticks. Also, the extensor carpi ulnaris, flexor carpi radialis, and extensor carpi radialis muscles are also useful in analyzing the holding up of drumsticks. These muscle relationships are, however, obtained from a qualitative viewpoint, so the actual relationships between them have not been reported. We tried to confirm the relationships by measuring the four muscle parts under each of the following conditions: the intended down stroke, intended up stroke, maintaining position, forced down stroke, and forced up stroke. Because the drummers need to strain arm muscles to control drumsticks, it is appropriate to measure the EMG of arms during drumming. Moreover, we can confirm which muscle parts described above are strained during drumming.

\subsection{Method}

In this measurement, the EMG signal and metronome signal were used, where the metronome signal showed the standards for downward and/or upward motion of the drumstick. An intermediate player participated in this test as the subject. The subject was asked to use a drumstick and his right arm was measured. The investigation procedure is as follows.

(a) Measurement of maintaining position. The subject was asked to hold the drumstick for 15 seconds in a consistent position, and the EMG in this situation was
Table 2 Experimental conditions.

\begin{tabular}{|c|c|c|c|c|}
\hline \multirow[b]{3}{*}{$\begin{array}{l}\text { Intended } \\
\text { motion }\end{array}$} & Up stroke & Down stroke & \multirow[b]{3}{*}{$\begin{array}{c}\text { Motion of } \\
\text { standard }\end{array}$} & \multirow{2}{*}{$\begin{array}{l}\text { Maintaining } \\
\text { position }\end{array}$} \\
\hline & \multirow{2}{*}{$\underbrace{}_{\substack{\text { player } \\
\text { VU }}} 1$} & & & \\
\hline & & $\begin{array}{l}\text { player } \\
\text { VD }\end{array}$ & & $\bigcup_{\text {player }} \rightarrow$ \\
\hline \multirow{3}{*}{ Forced motion } & \multirow{3}{*}{$\int_{\text {player }} 1$} & \multirow{3}{*}{$\bigoplus_{\text {player }}=0$} & & B \\
\hline & & & Length & $15 \mathrm{~s}$ \\
\hline & & & & \\
\hline \# of trials & 32 & 32 & & \\
\hline $\begin{array}{l}\text { Region of motion } \\
\text { of drumstick }\end{array}$ & \multicolumn{2}{|c|}{$\begin{array}{l}\text { Larger than } 25 \mathrm{~cm} \\
\text { for one motion }\end{array}$} & & \\
\hline
\end{tabular}

recorded. This is called basic (B) motion and was the standard to which other motions were compared.

(b) Measurement of intended motion. The subject was asked to play 32 single strokes, and the EMG signal was recorded during the strokes. The intended up stroke is called voluntary up (VU) stroke, and the intended down stroke voluntary down (VD) motion. When performing VU or VD motion, the subject was asked to stroke the drumstick up or down as in a real performance. All the motions were synchronized to the sound of a metronome. The range of drumstick motion was limited to within $25 \mathrm{~cm}$ between the highest and lowest point of the drumstick.

(c) Measurement of forced motion. The subject was asked to relax as much as possible while he gripped the drumsticks. His arms were forcibly moved to imitate the intended up and down motions, and the EMG signals of both of the arms were recorded 32 times. The motion of forced up stroke is called forced up (FU) stroke, and that of forced down stroke forced down (FD) stroke.

In this investigation, steps (a)-(c) were conducted. Experimental conditions of this investigation are shown in Table 2.

\subsection{Method of Analysis}

The four muscle parts described above were analyzed. A $50 \mathrm{~Hz}$ low-cut filter and a $500 \mathrm{~Hz}$ high-cut filter were prepared, and after this, full-wave rectification was conducted. For the EMG signal for B motion, represented as $m(t)$, the first and last $2500 \mathrm{~ms}$ were discarded and the remainder was used for analysis. The average EMG signal was then obtained.

To analyze the signals for VU, VD, FU, and FD motions, the range of time for stroking up or down was obtained by observing the metronome signal, and then each of the signals for VU, VD, FU, or FD motion was obtained, where the time was set to $500 \mathrm{~ms}$, and each duration was $500 \mathrm{~ms}$ long for each $j$ th region $(j=0,1, \cdots, 31)$. Specif- 


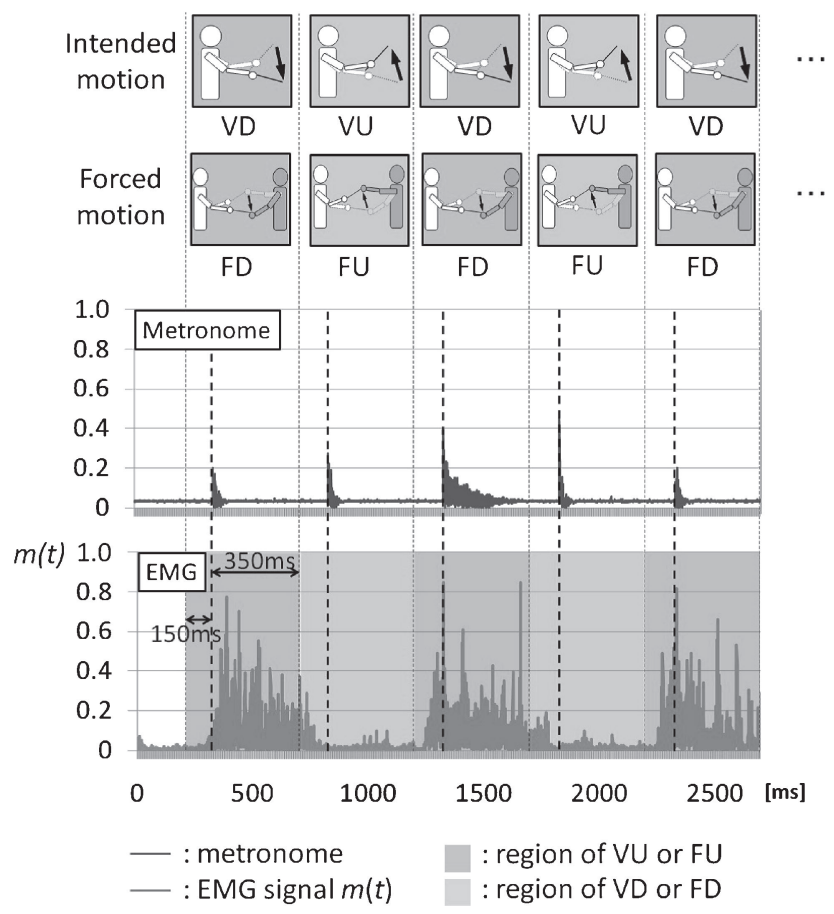

Fig. 8 Extraction of $m(t)$ for VU, VD, FU, and FD motions.

ically, the region of each motion was obtained from $t_{j}-$ 150 to $t_{j}+350 \mathrm{~ms}$ where $t_{j}$ represents the time of the metronome. Then the sum of signals $d_{j}$ was obtained, where the sampling rate was $2,000 \mathrm{~Hz}$, as

$$
d_{j}=\int_{t_{j}-150}^{t_{j}+350} m(t) d t
$$

The average $d_{j}$, called $P_{\text {action }}$, was then obtained, as

$$
P_{\text {action }}=\frac{\sum_{j=0}^{31} d_{j}}{32} .
$$

The outline of calculating EMG signals for VU, VD, FU, and FD motions is shown in Fig. 8.

Using $P_{x}$ for $x=$ basic, action obtained in Sect. 4.3, we investigate the appropriateness for the four muscle parts to correspond to drumming. As described in Sect. 4.1.2, the flexor carpi ulnaris, extensor carpi ulnaris, and flexor carpi radialis muscles are at work in stroking down. Therefore, for these muscles, a $t$-test for significant difference $(p<0.05)$ was conducted for the average scores between VD and other motions. On the other hand, the extensor carpi ulnaris muscle, flexor carpi radialis, and extensor carpi radialis muscles are at work in stroking up. Therefore, for these muscles, a test for significant difference $(p<0.05)$ was conducted for the average scores between VD and other motions.

\subsection{Results and Discussion}

Results of the test are shown in Table 3, and those for
Table 3 Results of significant difference test.

\begin{tabular}{|c|c|c|c|c|}
\hline & $V D>V U$ & $V D>F D$ & $V D>F U$ & $V D>B$ \\
\hline $\begin{array}{c}\text { Flexor carpi } \\
\text { ulnaris muscle }\end{array}$ & $* *$ & $* *$ & $* *$ & $* *$ \\
\hline $\begin{array}{c}\text { Extensor carpi } \\
\text { ulnaris muscle }\end{array}$ & $* *$ & $* *$ & $* *$ & $* *$ \\
\hline $\begin{array}{c}\text { Flexor carpi } \\
\text { radialis muscle }\end{array}$ & $* *$ & $* *$ & $* *$ & $* *$ \\
\hline & VU > VD & $V U>F U$ & $V U>F D$ & $V U>B$ \\
\hline $\begin{array}{c}\text { Extensor carpi } \\
\text { ulnaris muscle }\end{array}$ & - & - & $* *$ & $* *$ \\
\hline $\begin{array}{c}\text { Flexor carpi } \\
\text { radialis muscle }\end{array}$ & - & $* *$ & $* *$ & $* *$ \\
\hline $\begin{array}{c}\text { Extensor carpi } \\
\text { radialis muscle }\end{array}$ & $* *$ & $* *$ & $* *$ & $* *$ \\
\hline \\
$\left(^{*}: p<.05, * *: p<.01,-:\right.$ not significant) \\
\# of cases for VD, VU, FD, and FU: 32 \\
\# of cases for B: 20
\end{tabular}

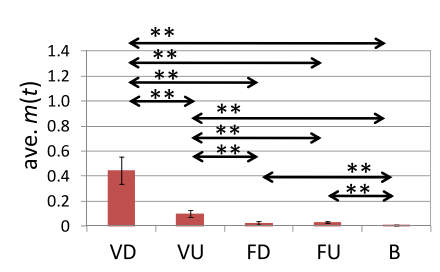

(a) Flexor carpi ulnaris muscle

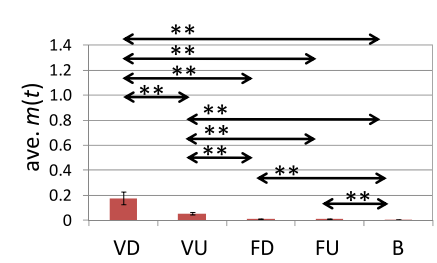

(c) Flexor carpi radialis muscle

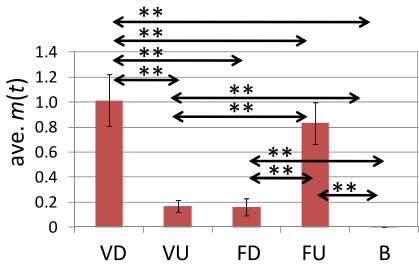

(b) Extensor carpi ulnaris muscle

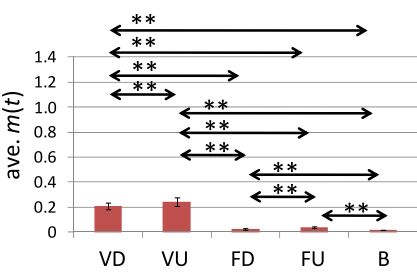

(b) Extensor carpi radialis muscle
Fig. 9 Obtained data of differences in average EMG for an intermediate player.

comparison are shown in Fig. 9. Concretely, in Table 3, the results of significant differences in comparisons of the amounts of muscle activities between VD and other motions are shown. Moreover, obtained data of differences in the average EMG signal for an intermediate player is shown in Fig. 9. From here, we discuss the test results.

For the flexor carpi ulnaris muscle, as shown in Table 3 and Fig. 9(a), the average EMG signal of VD motion was found to be much higher than those of other motions. As described in Sect. 4.1.1, the flexor carpi ulnaris muscle is thought to correspond to stroking down.

For the extensor carpi ulnaris muscle, as shown in Table 3 and Fig. 9(b), the average EMG signal of VD motion was found to be much higher than those of other motions. As described in Sect. 4.1.1, the extensor carpi ulnaris muscle is thought to correspond to stroking down, which is consistent with the test results. Moreover, the average score of VU motion was found to be much higher than that of $\mathrm{B}$ motion, whereas those for $\mathrm{VD}$ and $\mathrm{FU}$ 
motions are much higher than that of VU motion, which contradicts the description in Sect. 4.1.1.

For the flexor carpi radialis muscle, as shown in Table 3 and Fig. 9(c), the average EMG signal of VD motion was found to be much higher than those of others, which is consistent with the results in Sect. 4.1.1. Moreover, the average EMG signal of VU motion is much higher than those of FD, FU and B motions, and less than that of VD motion, showing that this muscle corresponds to stroking down.

For the extensor carpi radialis muscle, as shown in Table 3 and Fig. 9(d), the average score of EMG signals on VU motion was found to be much higher than those of other motions, which is consistent with the results in Sect. 4.1.1.

To summarize this investigation, the flexor carpi ulnaris, extensor carpi ulnaris, and flexor carpi radialis muscles were found to correspond to stroking down. Thus these muscles are appropriate for investigating the rebound feature. Moreover, the flexor carpi radialis and extensor carpi radialis muscles were found to correspond to stroking up. Thus these muscles are appropriate for investigating the motion of stroking up. However, the extensor carpi ulnaris muscle is strained for not only VU but also VD and FU motions, showing that it corresponds to $\mathrm{VU}$ and VD motions and somewhat to FU motion. As well, the flexor carpi radialis muscle corresponds to VU and VD motions, not to FU, FD or B motion, so we confirm the relationship between each muscle and drumming. Acocording these results, we will measure the four parts of muscles to measure the physical load of arms.

\section{RELATIONSHIP BETWEEN REBOUND FEATURE AND DRUMMING}

\subsection{Purpose}

As described in Sect. 1, the differences in the constitution and material affect the force or rebound. The force of a rebounding drumstick may affect the physical load of drumming [1]. There is thought to be a relationship between the differences in constitution as well as materials and the amount of physical load. As described in Sect. 2.2, the effect of the rebound feature on the physical load may change owing to the differences in ISI and hitting intensity. As also described in Sect. 3, the differences in constitution and material can be represented by the rebound coefficient. Moreover, as described in Sect. 4, the physical load of drumming can be represented by using the EMG signal.

Therefore, we conducted an experiment to investigate the playability of snare drums with different rebound features by controlling the five types of ISI and two types of intensity. Investigating the relationship between EMG signals and the coefficient of rebound was expected to clarify the playability of snare drums.
Table 4 Outline of snare drums.

\begin{tabular}{|c|c|c|c|c|}
\hline $\begin{array}{c}\text { Type of } \\
\text { snare drum }\end{array}$ & Name & $\begin{array}{l}\text { Material of } \\
\text { drumhead }\end{array}$ & $\begin{array}{l}\text { Shape of } \\
\text { drumhead }\end{array}$ & $\begin{array}{l}\text { Rebound } \\
\text { Coefficient }\end{array}$ \\
\hline $\begin{array}{l}\text { Acoustic } \\
\text { drum }\end{array}$ & $\begin{array}{c}\text { Acoustic snare } \\
\text { drum (AD) }\end{array}$ & $\begin{array}{c}\text { Polyester } \\
\text { film }\end{array}$ & \multirow{2}{*}{ Film } & 0.53 \\
\hline \multirow{3}{*}{$\begin{array}{l}\text { Electric } \\
\text { drum }\end{array}$} & $\begin{array}{l}\text { Electric snare } \\
\text { drum } 1 \text { (ED1) }\end{array}$ & Mesh & & 0.54 \\
\hline & $\begin{array}{l}\text { Electric snare } \\
\text { drum } 2 \text { (ED2) }\end{array}$ & Rubber pad & \multirow{2}{*}{ Pad } & 0.75 \\
\hline & $\begin{array}{l}\text { Electric snare } \\
\text { drum } 3 \text { (ED3) }\end{array}$ & Silicon pad & & 0.89 \\
\hline
\end{tabular}

\subsection{Method}

\subsubsection{Target to be analyzed}

Table 4 outlines the snare drums used in this test. The signals used were those of the EMG and metronome, and hitting signals of the snare drum, where the metronome was used as the standard for playing and the hitting signal was the acoustic signal obtained through the microphone. The strain of the four muscles on each arm were measured. Ten drummers (five professional and five intermediate players) participated in this test. All the drummers were asked to use two drumsticks to perform the given task.

\subsubsection{Experimental conditions}

The EMG is known as the index of muscle strain, so by normalizing all the obtained EMG signals using the EMG signals on the maximum intended strain, we could compare among players and conditions, because all the obtained EMG signals were represented as a ratio with the maximum and were independent of the variability of strength when specified by musical symbols such as $m p$ and $m f$. We used two conditions, $40 \%$ and $70 \%$, to specify the strength, instead of musical symbols. For the condition of tempi, five conditions $(30,80,130,180$, and $240 \mathrm{bpm}$ ) were used for a specified musical score comprised of eighth notes for 4/4. The "Slow" tempo conditions were 30,80 , and $130 \mathrm{bpm}$, and "Fast" were 180 and $240 \mathrm{bpm}$. All the experimental conditions are shown in Table 5.

\subsubsection{Display system for spontaneous EMG signal}

As described in Sect. 5.2.2, to measure the intensity of EMG signals when playing, the spontaneous EMG signal should be fed back visually to the drummer. Here we constructed a system for displaying the intensity of EMG signals for the flexor carpi ulnaris muscle while drumming, using LabVIEW. Our original system was used to show the intensity of EMG signals to the drummers during training. Figure 10 shows an image of the system, which yields a sum of EMG, every $30 \mathrm{~ms}$ by summing up all the EMG signals between each $30 \mathrm{~ms}$, and then it shows the results on the PC display for each arm. Drummers could easily see the intensity of EMG signals of their flexor carpi ulnaris muscles. 
Table 5 Experimental conditions.

\begin{tabular}{|c|c|c|}
\hline Task & Slow & Fast \\
\hline Score & $\begin{array}{l}\text { (16 measure) } \\
\text { (16 }\end{array}$ & $\begin{array}{c}\text { (8 measure) } \\
\text { (8)RLRLRLRL }\end{array}$ \\
\hline Tempo & $30 \mathrm{bpm}, 80 \mathrm{bpm}, 130 \mathrm{bpm}$ & $90 \mathrm{bpm}, 120 \mathrm{bpm}$ \\
\hline $\begin{array}{c}\text { Specified } \\
\text { amount of EMG }\end{array}$ & \multicolumn{2}{|c|}{$40 \%$ or $70 \%$ for the maximum contraction } \\
\hline
\end{tabular}

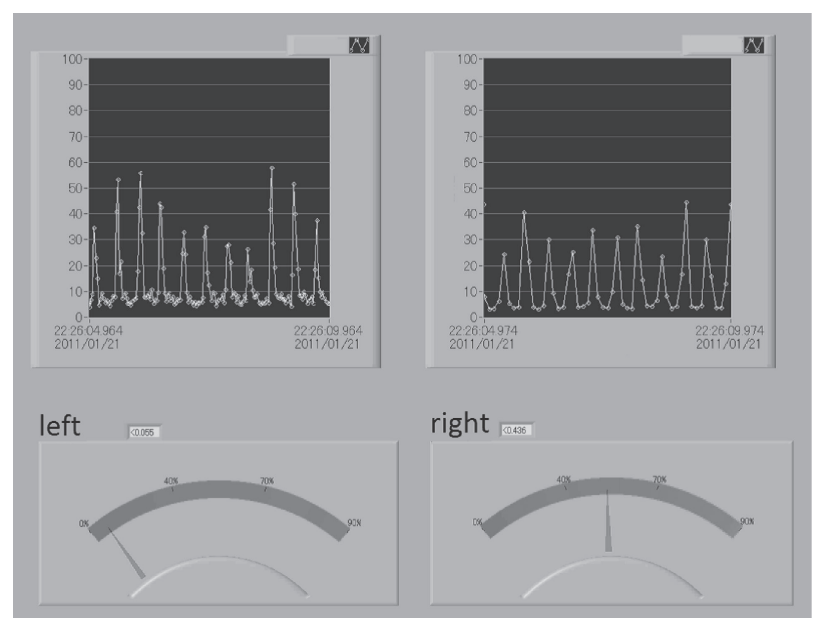

Fig. 10 Display system of spontaneous EMG signals.

\subsection{Flow of Measurement}

Below is the flow of the measurement.

(a) Each drummer was asked to perform volar flexion as strongly as possible, and the maximum strains on flexor carpi ulnaris and flexor carpi radials muscles were obtained as the standards for his/her measurement.

(b) Obtained EMG signals were calculated and represented as the ratio to the maximum amount obtained in (a). It was displayed every $30 \mathrm{~ms}$ in real time.

(c) Using the system, the player was asked to practice to be able to maintain the specified intensity of EMG signals without looking ats the spontaneous EMG.

(d) The drummer was asked to play a snare drum under a specified condition.

In this experiment, step (a) was conducted only once, and steps (b) and (d) were conducted for each trial. Ultimately, the combinations of experimental conditions were 4 (types of snare drum) $\times 5$ (types of tempo) $\times 2$ (intensities: $40 \%$ and $70 \%$ ) $=40$ patterns in total.

\subsection{Method of Analysis}

EMG signals were recorded under the flow described in Sect. 5.3. The four muscles were recorded for analysis. A $50 \mathrm{~Hz}$ low-cut filter and $500 \mathrm{~Hz}$ high-cut filter were prepared. After this, a full-wave rectification was con-

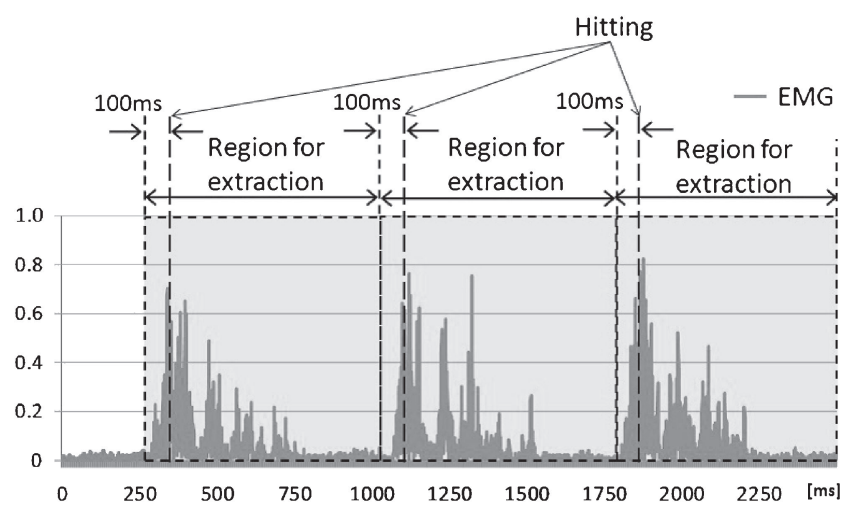

Fig. 11 Extraction of EMG signal for extensor carpi ulnaris muscle of left arm.

ducted, same as the described in Sect. 4.3. The region of stroking was obtained by using the metronome signal and hitting signal of the snare drum. The intensity of EMG signals became greater before $100 \mathrm{~ms}$ of hitting time, so the region between $100 \mathrm{~ms}$ before the current stroke and that before the next stroke is extracted. Figure 11 shows the extraction of each signal.

\subsection{Experimental Conditions}

The intensity of EMG signals for each stroke for all performances was obtained. To investigate the relationship between the rebound feature and EMG, the $t$-test for significant difference was conducted for each electric drum. Then the number of significantly different cases was determined. Concretely, the average amounts of EMG for each stroke between each electric snare drum and the acoustic snare drum, which had the lowest rebound coefficient, were compared by the $t$-test for significant difference. The test results revealed the ratio of significantly different cases. The cases compared were comprised of the level of players (professional or intermediate player), task, and parts to be measured. Under the "Slow" condition, there were 5 (players) $\times 3$ (tempo conditions) $\times 4$ (parts measured) $\times 2$ (left and right hands $)=120$ patterns in total. Under the "Fast" condition, there were 5 (players) $\times 2$ (tempo conditions) $\times 4$ (parts measured) $\times 2$ (left and right hands) $=80$ patterns in total. We then compared the number of significantly different cases among the 120 and 80 patterns for each electric snare drum.

\subsection{Results and Discussion}

Results for professional drummers are shown in Fig. 12, and those for intermediate drummers are shown in Fig. 13. The results of the comparison of the number of significantly different cases are given in Sect. 5.6.1, and the relationship between the rebound coefficient and the intensity of EMG signals is discussed in Sect. 5.6.2. 


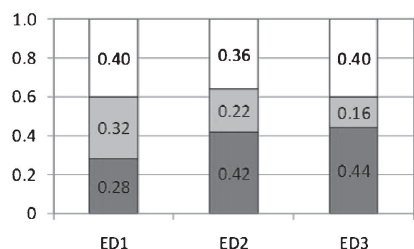

(a) Slow, $40 \%$

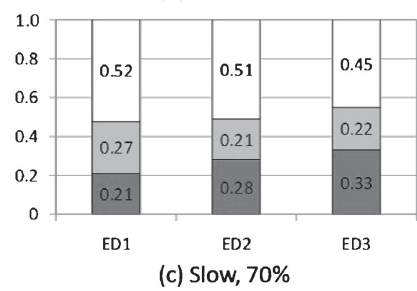

$\square$ :Significantly superior $\square$ : No significant difference

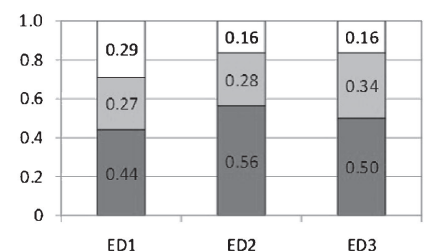

(b) Fast, $40 \%$

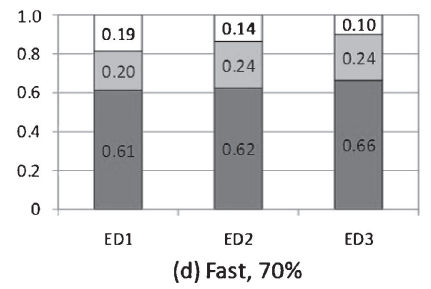

: Significantly inferior
Fig. 12 Ratio of the number of cases where EMG signal intensity is much higher than that for $\mathrm{AD}$ for five professional drummers.

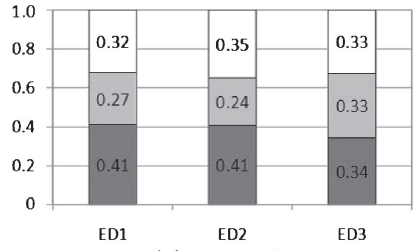

(a) Slow, $40 \%$

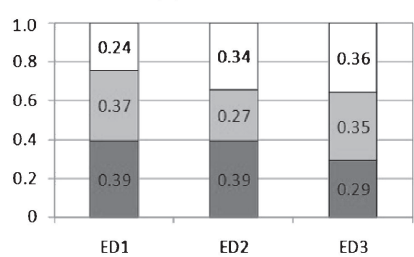

(c) Slow, $70 \%$

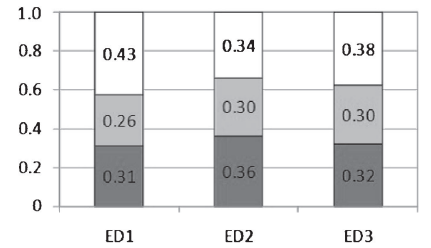

(b) Fast, $40 \%$

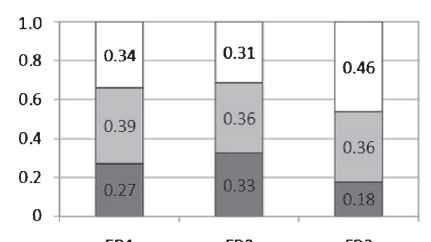

(d) Fast, $70 \%$ $\square$ :Significantly superior $\square$ : No significant difference $\square$ : Significantly inferior

Fig. 13 Ratio of the number of cases where EMG signal intensity is much higher than that for $\mathrm{AD}$ for five intermediate drummers.

\subsubsection{Results of comparison}

Figure 12 shows the ratio of the number of patterns for the intensity of EMG signals for the five professional drummers. As shown in Fig. 12(a), the values where the intensity of EMG signals is less than that for the $\mathrm{AD}$ increase the rebound coefficient under a Slow task and a weak intensity (in Fig. 12(a), 0.28, 0.42, 0.44). As shown in Fig. 12(b), the values where the intensity of EMG signals is more than that for the $\mathrm{AD}$ tend to decrease as the rebound coefficient increases under a Fast task and a weak intensity (in Fig. 12(b), 0.29, 0.16, 0.16). As shown in Fig. 12(c), the values where the intensity of EMG signals is less than that for the AD increase the rebound coefficient under a Slow task and a strong intensity (in Fig. 12(c), $0.21,0.28,0.33$ ). As shown in Fig. 12(d), the values where the intensity of EMG signals is less than that for the AD increases the rebound coefficient under a Fast task and a strong intensity (in Fig. 12(d), 0.61, 0.62, 0.66).

Figure 13 shows the ratio of the number of patterns for the intensity of EMG signals for the five intermediate drummers. No specific relationships can be found in Figs. 13(a) and 13(b). On the other hand, as shown in Fig. 13(c), the values where the EMG signal intensity is higher than those under the $\mathrm{AD}$ condition increase the value of the rebound coefficient $(0.24,0.34,0.36)$. No specific relationships can be observed in Fig. 13(d).

Moreover, we investigated the various tendencies among the professional and intermediate players. The results show that the variety of tendencies for the professional players is not marked, whereas that for intermediate players is relatively high. On the other hand, no difference is observed between the results for dominant and nondominant hands among professional and intermediate drummers.

\subsubsection{Results of comparison}

The results reveal that for professional drummers under all conditions, the electric snare drums with high rebound coefficients suppress the physical load of drumming more than those with low rebound coefficients. This result is confirmed to be common for four professional drummers. Thus, the electric snare drum with a high rebound coefficient is appropriate for professional drummers. On the other hand, for the intermediate drummers, no specific results were confirmed in terms of tendency among players. Moreover, a high variety among players was apparent in the individual results. Therefore, this implies that intermediate drummers cannot take into account the rebound of drumsticks although the professionals can.

On the other hand, the previous study showed the differences between skilled and unskilled drummers [9]. The study showed that the difference between skilled and unskilled drummers is apparent in the timing of the myoelectric activity on the extensor and flexor muscles of the wrist joint. The skilled drummers showed significantly earlier myoelectric activity of both the extensor and flexor muscle than the unskilled drummers. Concretely, the onset time of extensor muscle motion of skilled drummers was shown to be before impact, whereas that for unskilled drummers was after impact. The professional drummers in this study can control the drumstick by considering the effect of rebound, and they can do so in prior to impact, whereas intermediate players cannot. In general, drummers need to carry out preparation motion when drumming [10], so the extensor muscle motion corresponds to this preparatory motion.

Finally, we found that only the professional drummers can consider the strength of rebound when carrying out the preparatory motion of drumming. Moreover, we found that the electric snare drum with a high rebound feature can be 
effectively played by professional drummers but not by intermediate drummers in terms of physical loads.

\section{CONCLUSION}

In this study, we clarified the playability of snare drums by using their rebound features and physical load. The rebound coefficient was introduced as the index for the differences between snare drums, and the EMG signal was obtained to represent the physical load on the drummer's arms. First, we measured the rebound coefficient on the drumhead of the snare drum. Next, we examined the appropriateness of using EMG signals for analyzing drumming. Moreover, we measured the EMG signals for different types of snare drums. According to the results, the snare drum with high rebound is effectively played only by professional drummers, implying that only the professional drummers can take advantage of the rebound feature of each snare drum. Future tasks are to investigate drumming by considering becoming accustomed to drumming when playing for a long time and to use recorded videos and acoustic signals.

\section{ACKNOWLEDGEMENTS}

The author is grateful to Mr. Takuma Umehara and Ms. Yuki Konishi for their assistance in conducting measurements. This study is partly supported by a Grant-in-Aid for Scientific Research (22700112).

\section{REFERENCES}

[1] T. Fujisawa and M. Miura, "Investigating a playing strategy on drumming using surface electromyograms," Acoust. Sci. \&
Tech., 31, 300-303 (2010).

[2] R. Montes, M. Bedmar and M. S. Matin, "EMG biofeedback of the abductor pollicis brevis in piano performance, biofeedback and self-regulation," Appl. Psychophysiol. Biofeedback, 18, 67-77 (1993).

[3] B. Achermann, R. Adams and E. Marshall, "The effect of scapula taping on electromoygraphic actibity and mucical performance in professional violinist," Aust. J. Physiother. 2002, 48, 197-203 (2002).

[4] Roland Corp., "Roland V-Drum catalog 2010 Vol. 1" (2010) (in Japanese).

[5] Yamaha Corp., DTX-Series (2010).

[6] T. Kizuka, T. Masuda, T. Kiryu and T. Sadoyama, Electromyogram (Tokyo Denki University Press, Tokyo, 2006) (in Japanese).

[7] Y. Nagano, PLAYERS' HANDBOOOKS All Knowledge for Drummer (Ritto Music, Tokyo, 1995) (in Japanese).

[8] A. O. Perotto, Anatomical Guide for the Electromyographer: The Limbs and Trunk, Translated verision (Nishimura-Syoten, Niigata, 1997) (in Japanese).

[9] K. Nishimura and C. Matsubara, "Characteristics of the stickwork structure during snare drumming," Sci. Rep. Kyoto Pref. Univ., Hum. Env. Agr., 59 1-9 (2007).

[10] S. Dahl, "Arm motion and striking force in drumming," Proc. Int. Symp. Musical Acoustics 2001, pp. 293-296 (2001).

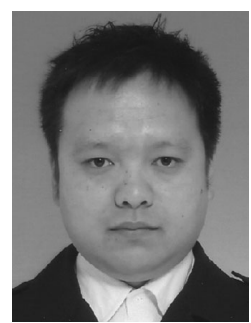

Masanobu Miura received B.E., M.E. and $\mathrm{Ph} . \mathrm{D}$ degrees in Engineering from Doshisha University, Japan, in 1998, 2000 and 2003, respectively. In 2003, he joined Department of Media Informatics, Faculty of Science and Technology, Ryukoku University and is currently an associate professor. He received the Awaya-Kiyoshi Award and the Sato Paper Award, in 2002 and 2004, respectively. He is a member of ASJ, JSMPC, IPSJ, IEICE, JPA and ASA. 